was commenced on paroxetine $30 \mathrm{mg}$ during the sixth month of pregnancy, because of increased depressive symptoms, restlessness and decreased appetite.

A male neonate $(4160 \mathrm{~g})$ was delivered vaginally at 39 weeks' gestation after a normal pregnancy. The delivery was without complications. The newborn initially appeared alert (Apgar 9-10-10). At the age of 12 hours he was transferred to the neonatal department for observation because of increased respiratory rate $(80$ per minute) and jitteriness. During the next hours he developed increased muscle tone and tremor. When 'pulled-to-sit', there was a stiff flex in the elbows and the head was flexed forwards. There were no convulsions. The child was bottle-fed with formula without problems. C-reactive protein, haemoglobin, blood gases, blood glucose, electrolytes, ionised calcium and ultrasound of the brain were all normal. The symptoms decreased successively during the third and fourth day and the child was discharged at the age of four days with a normal neurological status except for sustained jitteriness. A follow-up at the age of four weeks showed a fully normal neurological status.

The serum paroxetine level in the child was $68 \mathrm{nmol} / 1$ at the age of one day, $75 \mathrm{nmol} / \mathrm{h}$ at two days and $23 \mathrm{nmol} / \mathrm{l}$ at three days of age. No serum or breast milk paroxetine concentrations were available from the mother, who chose not to breastfeed because of the drug treatment and earlier negative experience with breastfeeding. The mother's serum paroxetine concentration was $185 \mathrm{nmol} / 125$ days after delivery, during continued treatment with $30 \mathrm{mg}$ paroxetine per day.

We have been able to locate only two earlier reports of neonatal toxicity after maternal treatment with SSRIs; one for fluoxetine (Spencer, 1993) and one for sertraline (Kent \& Laidlaw, 1995), with similar findings as in the present case. Poor neonatal adaptation with respiratory difficulties, cyanosis on feeding and jitteriness, were also associated with third-trimester exposure to fluoxetine in a recent study (Chambers et al, 1996). It is usually recommended that treatment with tricyclic antidepressants should be stopped or the dosage decreased before delivery to avoid neonatal withdrawal syndrome (Briggs et al, 1994). As very little information is available for the newer antidepressants, including SSRIs, they are not recommended during pregnancy. The increased popularity of these drugs in the treatment of various psychiatric disorders will, however, eventually lead even to pregnant women being exposed. The risk of neonatal withdrawal syndrome should in such cases be acknowledged, and dose reduction before delivery considered.

Brizes, G. G., Freeman, R. K. \& Yaffo, S. J. (eds) (1994) Drugs in Pregnoncy and Loctotion (4th edn). pp. 192-195. Baltimore. MD: Williams \& Wilkins.

Chambers, C. D., Johmson, K. A., Dick, L. M., et al (1996) Birth outcomes in pregnant women taking fluoxetine. New England fournal of Medicine, 335, 1010-1015.

Kent, L. S. W. \& Labdiaw, J. D. D. (1995) Suspected congenital sertraline dependence (letter). British journol of Psychiatry, 167, 412-413.

Spencer, M. J. (1993) Fluoxetine hydrochloride (Prozac) toxicity in a neonate. Pediatrics, $92,721-722$

M. L. Dahl Department of Medical Laboratory Sciences \& Technology, Division of Clinical Pharmacology. Huddinge University Hospital, Huddinge. Sweden

E. Olhager Department of Pediatrics, University Hospital, Linköping, Sweden

J. AhIner Department of Clinical Pharmacology. University Hospital, Linköping, Sweden

\section{Subjective quality of life in schizophrenia}

Sir: Franz et al (1997) discuss in their paper that atypical neuroleptics may have subjective quality of life advantages over conventional antipsychotics. Although their assertion may well prove to be true in the future, once we have more robust studies on this subject, I am not sure their conclusions are justified by the results of their study.

They applied the Munich Quality of Life Dimensions List (MLDL-GI) to a group of in-patients on day 10 of admission. They compared scores in those receiving conventional antipsychotics with those on atypical antipsychotics, and found the latter to have a significantly higher general quality of life. My first point is that they do not seem to have administered a baseline MLDL-GI (i.e. prior to commencing antipsychotics), so we do not know whether the higher scores in the group receiving atypical antipsychotics can be attributed to the medication. It could be possible that this group had higher scores to begin with. Second, the four subscales of the MLDL-GI include physical domain, mental domain, social life and everyday life. Although it may be valid to measure the physical and mental domain of in-patients 10 days after admission, I am not sure about the validity of measuring social life and everyday life in a group of in-patients recently admitted to hospital. Surely, social and everyday life must be measured within the context of the patient's own home and environment.

Franz, M., Lis, S., Pluddemann, K., et ol (1997) Conventional versus atypical neuroleptics: subjective quality of life in schizophrenic patients. British journal of Psychiatry 170, 422-425

M. R. Agarwal Ferndale Unit, Fazakerley Hospital, Liverpool L9 7AL

Author's reply: The study to which $\mathrm{Dr}$ Agarwal refers was an open pilot study based on a non-experimental crosssectional design. Assessment of subjective quality of life (SQoL) at baseline would be beyond the scope of such an explorative study. Restrictions to open studies have been discussed in the paper and the findings are, therefore, tested using an experimental double-blind repeated-measures design in a study that has just started. We definitely agree that different patients have different individual levels of satisfaction, which may influence their SQoL ratings.

The quality of life inventory is constructed in such a way that it can be used in whatever circumstances patients live. Hospital wards sometimes offer more of a 'home' to patients than the outside situation. Therefore, it is our opinion that validity of the assessment of SQoL in the domains of social life and everyday life does not depend on the place the patient is staying at the time. A patient always has a social and an everyday life. There are fellow patients, the hospital staff or visitors within the hospital, or there are friends and relatives back home. The assessment becomes valid if it really reflects only SQoL and no other dimension within this area of concern. In our study, patients were asked to assess their SQoL in different aspects of their present interpersonal contacts (social life subscale). Whether or not the assessment in this situation is representative of their predominant way of living is another question. The purpose of the study, however, was to investigate the present SQoL of newly admitted in-patients, subsequent to a psychotic exacerbation and taking different kinds of antipsychotic medication, within an exploratory research design.

M. Franz Centre for Psychiatry, Justus-Liebig University, School of Medicine, Am Steg 22 . D-38385 Giessen, Germany 